\title{
Medicamentos cardiovasculares: prevalência e fatores associados em idosos da Estratégia Saúde da Família de Porto Alegre
}

\author{
Vanessa Machado de Azevedo*, Paula Engroff*, Vanessa Sgnaolin ***, Fernanda Loureiro***, \\ Camila Pereira de Andrade ${ }^{* * * *}$, Newton Luiz Terra ${ }^{* * * * * *}$, Irenio Gomes ${ }^{* * * * * *}$
}

\section{Resumo}

O objetivo foi investigar a prevalência do uso de medicamentos cardiovasculares e sua associação com variáveis sociodemográficas e clínicas em idosos atendidos na Estratégia Saúde da Família por meio de um estudo transversal realizado no município de Porto Alegre. A coleta de dados foi feita com um questionário que observou aspectos sociodemográficos, econômicos, relacionados a doenças autorreferidas e uso de medicamentos. Foram avaliados 761 idosos, que tiveram $67,7 \%$ de prevalência de uso de medicamentos cardiovasculares. As características que mostraram uma associação significativa, de forma independente, com o uso de alguma medicação cardiovascular foram: sexo feminino, cor negra, consulta nos últimos seis meses, hipertensão, diabetes e doença cerebrovascular. Os medicamentos cardiovasculares e as classes mais utilizadas, respectivamente, foram: hidroclorotiazida $(34,8 \%)$ e captopril (33,5\%); agentes que atuam sobre o sistema renina-angiotensina $(46,8 \%)$ e diuréticos (42\%). Esses dados são importantes, pois a população idosa é afetada sobretudo por doenças cardiovasculares, e dados populacionais podem auxiliar na formulação de estratégias e políticas públicas voltadas, principalmente, para a população idosa atendida pelo Sistema Único de Saúde.

Palavras-chave: Doenças cardiovasculares. Estratégia Saúde da Família. Fármacos. Idoso.

* Acadêmica no Curso de Farmácia, Faculdade de Farmácia, Pontifícia Universidade Católica do Rio Grande do Sul, Porto Alegre, Brasil. E-mail: nessinha_ma@msn.com

*** Doutora em Gerontologia Biomédica, Programa de Pós-Graduação em Gerontologia Biomédica, Pontifícia Universidade Católica do Rio Grande do Sul, Porto Alegre, Brasil. Farmacêutica. E-mail: paula_puc@yahoo. com.br

**** Doutoranda no Programa de Pós-Graduação em Gerontologia Biomédica, Pontifícia Universidade Católica do Rio Grande do Sul, Porto Alegre, Brasil. Farmacêutica. Endereço para correspondência: Laboratório de Bioquímica, Genética Molecular e Parasitologia do Instituto de Geriatria e Gerontologia, Hospital São Lucas da PUCRS, Avenida Ipiranga, 6690, $3^{\circ}$ andar, Bairro Jardim Botânico, 90610-000, Porto Alegre, RS. Tel.: (55 51) 3320-3000 (ramal: 2660). E-mail: vanessasgnaolin@yahoo.com.br

**** Pós-doutoranda no Programa de Pós-Graduação em Gerontologia Biomédica, Pontifícia Universidade Católica do Rio Grande do Sul, Porto Alegre, Brasil. Fonoaudióloga. E-mail: fernanda0801@gmail.com

****** Acadêmica no Curso de Farmácia, Faculdade de Farmácia, Pontifícia Universidade Católica do Rio Grande do Sul, Porto Alegre, Brasil. E-mail: camila.andrade.002@acad.pucrs.br

******* Médico geriatra. Diretor do Instituto de Geriatria e Gerontologia. Professor do Programa de Pós-Graduação em Gerontologia Biomédica, Pontifícia Universidade Católica do Rio Grande do Sul, Porto Alegre, Brasil. E-mail: terranl@pucrs.br

******** Médico neurologista. Professor do Programa de Pós-Graduação em Gerontologia Biomédica, Pontifícia Universidade Católica do Rio Grande do Sul, Porto Alegre, Brasil. E-mail: irenio@nienpe.org

$\rightarrow$ http://dx.doi.org/10.5335/rbceh.v14i2.6470

Recebido em: 25.10.2016. Aceito em: 06.12.2017. 


\section{Introdução}

De acordo com o censo brasileiro realizado em 2010 pelo Instituto Brasileiro de Geografia e Estatística (IBGE), as pessoas com 65 anos ou mais representavam $7,4 \%$ da população, enquanto que, no ano de 2000, a população idosa era de $5,9 \%$. Isso demonstra que o Brasil segue uma tendência que já vem ocorrendo em países desenvolvidos: o envelhecimento da população (IBGE, 2011). A proporção de idosos vem crescendo de forma acelerada, enquanto a proporção de crianças vem diminuindo (IBGE, 2002). As projeções para 2050 referem uma população brasileira de idosos maior do que a de crianças e adolescentes de 14 anos ou menos (MAFRA et al., 2013). Isso se deve a quedas nas taxas de fecundidade e mortalidade e ao aumento da expectativa de vida nos países em desenvolvimento (IBGE, 2002; WORLD HEALTH ORGANIZATION, 2005).

Envelhecer com qualidade de vida será o principal desafio da população e também dos serviços e dos profissionais de saúde. Estima-se que, em 2025, o Brasil alcançará o posto de sexto país no mundo em número de idosos, com um contingente superior a 30 milhões de pessoas (WORLD HEALTH ORGANIZATION, 2005). Devido a isso, projeta-se também um maior número de pessoas acometidas por doenças crônicas, sendo as doenças cardiovasculares uma das mais prevalentes entre os idosos (SANTOS JÚNIOR; OLIVEIRA; SILVA, 2014; JACKSON; WENGER, 2011). Isso resulta numa maior procura pelos serviços de saúde e no aumento do consumo de medicamentos por esse grupo etário.

Doenças cardiovasculares, como hipertensão arterial sistêmica (HAS), doença cardíaca coronariana, fibrilação atrial, insuficiência cardíaca e acidente vascular cerebral, são comumente relatadas em indivíduos idosos e vêm liderando as causas de morbimortalidade (YAZDANYAR; NEWMAN, 2009; CHEN et al., 2014). Dentre essas doenças, a HAS é considerada um dos mais importantes problemas de saúde pública e um dos principais fatores de risco modificáveis, com alta prevalência e baixas taxas de controle (SOCIEDADE BRASILEIRA DE CARDIOLOGIA, 2010). A mortalidade relacionada com doença cardiovascular aumenta progressivamente com a elevação da pressão arterial (PA). Conforme dados mundiais referentes a 2001, cerca de 7,6 milhões de mortes foram atribuídas à elevação da $\mathrm{PA}$, sendo $54 \%$ por acidente vascular encefálico e $47 \%$ por doença isquêmica do coração (WILLIAMS, 2010). As alterações cardiovasculares também são fatores de risco para o desenvolvimento de depressão e demências (JANI et al., 2014; KALARIA et al., 2008).

Os medicamentos cardiovasculares são amplamente prescritos para fins preventivos e terapêuticos (ALI RAZA; MOVAHED, 2002). Sendo assim, os medicamentos representam um dos itens mais importantes da atenção à saúde do idoso, pois estes são os maiores usuários de polifarmácia e, ainda, 
apresentam particularidades farmacocinéticas e farmacodinâmicas que levam a alterações fisiopatológicas dos sistemas cardiovascular, hepático e renal, tornando os idosos mais vulneráveis a efeitos adversos e interações medicamentosas, além de prejudicar também a adesão ao tratamento por essa população.

O monitoramento da terapia medicamentosa cardiovascular na população idosa é crucial para reduzir as complicações com o uso de medicamentos. Embora o Estatuto do Idoso (BRASIL, 2003) e a Política Nacional de Saúde da Pessoa Idosa (BRASIL, 1999) prevejam assistência integral e gratuita ao idoso pelo Sistema Único de Saúde (SUS), além do acesso a medicamentos e tratamento das doenças, esse monitoramento às vezes se torna deficiente na população de baixa renda. Segundo Viacava (2010), da população brasileira atendida pelo SUS, apenas $45 \%$ dos usuários adquirem todos os medicamentos gratuitamente por esse sistema. Isso ocorre devido à ausência de medicamentos na Unidade Básica de Saúde (UBS), à falta de adesão por parte dos usuários e à carência de uma orientação farmacêutica, já que tal profissional não está inserido em todas as farmácias das UBS.

A maioria dos estudos disponíveis na literatura descreve a utilização de medicamentos em idosos de uma forma geral (COELHO FILHO; MARCOPITO; CASTELO, 2004; DAMIÃO et al., 2011; FLORES; BENVEGNÚ, 2008; GOULART et al., 2014; RIBAS; OLIVEIRA, 2014), mas poucos são aqueles que dão ênfase aos cardiovasculares (ALI RAZA; MOVAHED, 2002), principalmente levando em consideração a população brasileira (PERROTTI et al., 2007; GONTIJO et al., 2012). Observa-se que existe uma carência de estudos de base populacional que forneçam uma avaliação ampla dos medicamentos cardiovasculares prescritos para idosos. Em vista disso, o objetivo do presente trabalho foi investigar a prevalência do uso de medicamentos cardiovasculares $\mathrm{e}$ sua associação com variáveis sociodemográficas e clínicas em idosos atendidos na Estratégia Saúde da Família (ESF) de Porto Alegre.

\section{Métodos}

\section{Delineamento}

Este estudo é transversal, descritivo e analítico, coletado de forma prospectiva em uma amostra aleatória da população de idosos cadastrados na ESF do município de Porto Alegre. Fez parte do projeto de pesquisa Estudo Epidemiológico e Clínico dos Idosos Atendidos pela ESF do Município de Porto Alegre (Emisus), que se desenvolveu no período de março de 2011 a dezembro de 2012 (GOMES et al., 2013).

O tamanho amostral para o estudo foi calculado utilizando um nível de significância de 0,05. Considerando uma população-alvo de 22.000 idosos atendidos pela ESF em Porto Alegre, foi definido um tamanho amostral mínimo de 758 idosos, considerando 3,5\% de erro aceitável para uma prevalência 
esperada de $50 \%$ a $70 \%$. Devido a possíveis perdas amostrais e à estrutura de coleta de dados, o estudo foi projetado para uma amostra aleatória de 1.080 idosos. Inicialmente, foram selecionadas, de forma aleatória e estratificada por regiões distritais, 30 equipes da ESF do município de Porto Alegre.

Após a seleção das equipes, os agentes comunitários de saúde (ACS) foram contatados, e cada equipe apresentou o nome de todos os usuários idosos matriculados em sua região, para o sorteio dos participantes, 36 indivíduos de cada uma das equipes. Antes da realização da coleta de dados, os pesquisadores orientaram os ACS sobre os objetivos do estudo e a capacitação acerca da aplicação do questionário geral de coleta de dados, elaborado pelos pesquisadores. Os critérios de inclusão foram idade igual ou superior a 60 anos, cadastro na ESF, realização de entrevista completa com os ACS.

A pesquisa foi composta por uma etapa inicial de triagem e coleta de dados gerais, em que os ACS coletaram dados relacionados a aspectos sociodemográficos, econômicos e sociais, doenças autorreferidas e uso de medicamentos. Posteriormente, avaliações especializadas foram realizadas no Hospital São Lucas da Pontifícia Universidade Católica do Rio Grande do Sul (HSL/PUCRS) por uma equipe multidisciplinar, que verificou aspectos clínicos, nutricionais e físicos dos idosos.

As variáveis estudadas compreendem: dados sociodemográficos e clínicos (sexo, idade, estado civil, raça, escolaridade, renda individual, condições de saúde, PA, índice de massa corporal (IMC) e utilização de medicamentos cardiovasculares). A informação quanto ao uso de medicamentos foi coletada pelo agente comunitário de saúde em visita domiciliar, considerando os medicamentos que o indivíduo estava utilizando naquele momento com ou sem prescrição. Os medicamentos foram identificados de acordo com o seu princípio ativo e, após, classificados de acordo com o Sistema de Classificação Anatômico, Terapêutico e Químico (ATC), a partir de consulta à página eletrônica da Organização Mundial da Saúde (OMS). Foi considerado como cardiovascular o medicamento classificado como "C" e a classe farmacológica pelo respectivo subnível (WORLD HEALTH ORGANIZATION, 2015).

A PA foi mensurada posteriormente, na avaliação médica especializada, e classificada como normal $(<140 / 90$ $\mathrm{mmHg})$ ou alterada $(\geq 140 / 90 \mathrm{mmHg})$, segundo recomendações da Sociedade Brasileira de Cardiologia (SBC) (2010).

As variáveis para o cálculo do IMC (peso em $\mathrm{kg}$ e altura em metros) foram medidas pelas nutricionistas também na avaliação multiprofissional realizada no HSL/PUCRS. O IMC foi classificado segundo a OMS (WORLD HEALTH ORGANIZATION, 2000), considerando obesidade IMC $\geq 30$; sobrepeso IMC $\geq 25$ e $<30$; eutrófico IMC $\geq 18,5$ e $<25$; baixo peso IMC $<18,5$. 
Os dados foram analisados por meio do programa estatístico SPSS, versão 17. As variáveis foram descritas por meio de frequências, médias e desvios padrões. $\mathrm{Na}$ análise bivariada, foi utilizado o teste Qui-Quadrado de Pearson, que foi empregado para testar a associação entre as variáveis categóricas. As variáveis ordinais foram comparadas pelo teste de tendência linear do Qui-Quadrado. Os resultados foram considerados significativos quando $P<0,05$. Na análise multivariada, foi utilizada a regressão de Poisson com o objetivo de avaliar as variáveis que se associaram de forma independente.

Este projeto foi aprovado pelos Comitês de Ética em Pesquisa da PUCRS (Parecer $n^{\circ}$ 10/04967) e da Secretaria Municipal de Saúde da Prefeitura do Município de Porto Alegre (Processo $\mathrm{n}^{\circ}$ 001.021434.10.7), atendendo às Diretrizes e Normas Regulamentadoras em Pesquisa, conforme a Resolução no 196/1996 do Conselho Nacional de Saúde, vinculado ao Ministério da Saúde. O termo de consentimento livre e esclarecido foi assinado por todos os participantes do estudo.

\section{Resultados}

Dos 1.080 idosos sorteados para participar da pesquisa, 319 não foram localizados (tinham mudado de endereço ou ido a óbito). O grupo estudado foi composto por 761 participantes, com idade entre 60 e 103 anos e média de $69,0 \pm 7,3$ anos. A maioria era do sexo feminino $(63,7 \%)$, da cor branca $(64,7 \%)$, com ensino fundamental incompleto $(66,6 \%)$ e com renda de até um salário mínimo $(65,7 \%)$. A prevalência do uso de medicamentos cardiovasculares foi de $67,7 \%$, sendo que o sexo feminino, a faixa etária entre 70 e 79 anos e a cor negra apresentaram uma frequência de uso significativamente maior (Tabela 1). 
Tabela 1 - Frequência de uso de medicamento cardiovascular associado com variáveis sociodemográficas em idosos da Estratégia Saúde da Família de Porto Alegre

\begin{tabular}{|c|c|c|c|}
\hline Variáveis sociodemográficas & $\begin{array}{c}\text { População } \\
\text { n (\%) }\end{array}$ & $\begin{array}{c}\text { Frequência de uso de } \\
\text { medicamento } \\
\text { cardiovascular } \\
\%\end{array}$ & $P$ \\
\hline \multicolumn{4}{|l|}{ Sexo } \\
\hline Feminino & $485(63,7)$ & 73,4 & \multirow{2}{*}{$<0,001^{\#}$} \\
\hline Masculino & $276(36,3)$ & 57,6 & \\
\hline \multicolumn{4}{|l|}{ Idade } \\
\hline $60-69$ anos & $448(59,2)$ & 63,6 & \multirow{3}{*}{$0,032^{\varepsilon}$} \\
\hline 70-79 anos & $235(31,0)$ & 75,7 & \\
\hline 80 anos ou mais & $74(9,8)$ & 67,6 & \\
\hline \multicolumn{4}{|l|}{ Cor } \\
\hline Branca & $486(64,7)$ & 66,3 & \multirow{4}{*}{$0,050^{\#}$} \\
\hline Parda & $98(13,0)$ & 61,2 & \\
\hline Negra & $145(19,3)$ & 76,6 & \\
\hline Outras & $22(3,0)$ & 72,7 & \\
\hline \multicolumn{4}{|l|}{ Estado civil } \\
\hline Solteiro & $134(17,7)$ & 60,4 & \multirow{4}{*}{$0,084^{\#}$} \\
\hline Casado & $279(36,9)$ & 67,0 & \\
\hline Separado & $111(14,7)$ & 66,7 & \\
\hline Viúvo & $232(30,7)$ & 73,3 & \\
\hline \multicolumn{4}{|l|}{ Escolaridade } \\
\hline Analfabeto & $136(18,2)$ & 61,0 & \multirow{3}{*}{$0,080^{£}$} \\
\hline Ensino fundamental incompleto & $498(66,6)$ & 68,9 & \\
\hline Ensino fundamental completo ou mais & $114(15,2)$ & 71,1 & \\
\hline \multicolumn{4}{|l|}{ Renda } \\
\hline Nenhuma & $59(8,1)$ & 64,4 & \multirow{4}{*}{$0,931^{£}$} \\
\hline 1 salário mínimo & $417(57,6)$ & 67,6 & \\
\hline 2 salários mínimos & $191(26,4)$ & 69,6 & \\
\hline 4 salários mínimos ou mais & $57(7,9)$ & 61,4 & \\
\hline Total & $761(100,0)$ & 67,7 & \\
\hline
\end{tabular}

Fonte: primária.

Legenda: \# Teste Qui-Quadrado de Pearson. ${ }^{£}$ Teste de Tendência Linear do Qui-Quadrado.

Nota: o total de cada variável pode não atingir o n de 761 devido a perdas amostrais. 
Os dados clínicos e de saúde são apresentados na Tabela 2. Foi observado que quanto maior a quantidade de medicamentos utilizados, maior a prevalência do uso de cardiovasculares $(P<$
0,001). Os idosos que tiveram consulta médica nos últimos seis meses, aqueles com maior IMC e PA sistólica alterada também relataram utilizar mais medicamentos cardiovasculares.

Tabela 2 - Frequência de uso de medicamento cardiovascular associado com variáveis clínicas e de saúde em idosos da Estratégia Saúde da Família de Porto Alegre

\begin{tabular}{|c|c|c|c|}
\hline Variáveis clínicas e de saúde & $\begin{array}{c}\text { População } \\
\text { n (\%) }\end{array}$ & $\begin{array}{c}\text { Frequência de uso } \\
\text { de medicamento } \\
\text { cardiovascular } \\
\%\end{array}$ & $\boldsymbol{P}$ \\
\hline \multicolumn{4}{|l|}{ Autopercepção de saúde } \\
\hline Ótima/Boa & $268(35,5)$ & 64,2 & \multirow{3}{*}{$0,353^{\varepsilon}$} \\
\hline Regular & $406(53,8)$ & 70,4 & \\
\hline Má/Péssima & $81(10,7)$ & 65,4 & \\
\hline \multicolumn{4}{|l|}{ Cuidador } \\
\hline Não & $444(60,8)$ & 66,2 & \multirow{2}{*}{$0,148^{\#}$} \\
\hline Sim & $286(39,2)$ & 71,3 & \\
\hline \multicolumn{4}{|l|}{ Quantidade de medicamentos* } \\
\hline 0 & $113(14,8)$ & 0 & \multirow{4}{*}{$<0,001^{£}$} \\
\hline $1-2$ & $149(19,6)$ & 47,7 & \\
\hline $3-5$ & $281(36,9)$ & 84,0 & \\
\hline 6 ou mais & $218(28,6)$ & 95,4 & \\
\hline \multicolumn{4}{|l|}{ Consulta médica (últimos 6 meses) } \\
\hline Não & $101(13,4)$ & 40,6 & \multirow{2}{*}{$<0,001^{\#}$} \\
\hline Sim & $653(86,6)$ & 72,0 & \\
\hline \multicolumn{4}{|l|}{$\mathrm{IMC}^{\&}$} \\
\hline Baixo peso & $9(1,6)$ & 22,2 & \multirow{4}{*}{$<0,001^{£}$} \\
\hline Eutrófico & $140(24,6)$ & 62,1 & \\
\hline Sobrepeso & $222(38,9)$ & 68,5 & \\
\hline Obesidade & $199(34,9)$ & 76,4 & \\
\hline \multicolumn{4}{|l|}{ PAS $^{\$}$} \\
\hline Normal & $319(57,9)$ & 65,2 & \multirow{2}{*}{$0,014^{\#}$} \\
\hline Alterada & $232(42,1)$ & 75,0 & \\
\hline \multicolumn{4}{|l|}{$\mathrm{PAD}^{\$}$} \\
\hline Normal & $346(62,8)$ & 67,1 & \multirow{2}{*}{$0,132^{\#}$} \\
\hline Alterada & $205(37,2)$ & 73,2 & \\
\hline
\end{tabular}

Fonte: primária.

Legenda: *Uso de medicamentos considerando todas as classes farmacológicas. \# Teste Qui-Quadrado de Pearson. ${ }^{£}$ Teste de Tendência Linear do Qui-Quadrado. \& Dados obtidos de 570 idosos que realizaram avaliação antropométrica. ${ }^{\$}$ Dados obtidos de 510 idosos que tiveram sua pressão arterial aferida. IMC = índice de massa corporal; PAS = pressão arterial sistólica; $\mathrm{PAD}=$ pressão arterial diastólica.

Notas: o total de cada variável pode não atingir o n de 761 devido a perdas amostrais. 
Os idosos que relataram HAS, in- vascular ou doença mental utilizavam suficiência cardíaca congestiva, doença mais medicamentos cardiovasculares vascular periférica, infarto agudo do mio- do que os indivíduos que não relataram cárdio, angina, diabetes, doença cerebro- essas doenças (Tabela 3).

Tabela 3 - Frequência de uso de medicamento cardiovascular associado com doenças em idosos da Estratégia Saúde da Família de Porto Alegre

\begin{tabular}{|c|c|c|c|}
\hline Doenças & $\begin{array}{c}\text { População } \\
\text { n (\%) }\end{array}$ & $\begin{array}{c}\text { Frequência de uso de } \\
\text { medicamento cardiovascular } \\
\%\end{array}$ & $P$ \\
\hline \multicolumn{4}{|c|}{ ( } \\
\hline Não & $231(30,4)$ & 19,9 & \multirow{2}{*}{$<0,001^{\#}$} \\
\hline Sim & $530(69,6)$ & 88,5 & \\
\hline \multicolumn{4}{|l|}{ ICC } \\
\hline Não & $656(86,2)$ & 64 & \multirow{2}{*}{$<0,001^{\#}$} \\
\hline $\operatorname{Sim}$ & $105(13,8)$ & 90,5 & \\
\hline \multicolumn{4}{|c|}{ Doença vascular periférica } \\
\hline Não & $625(82,1)$ & 65 & \multirow{2}{*}{$0,001^{\#}$} \\
\hline Sim & $136(17,9)$ & 80,1 & \\
\hline \multicolumn{4}{|l|}{ IAM } \\
\hline Não & $671(88,2)$ & 65,6 & \multirow{2}{*}{$0,001^{\#}$} \\
\hline Sim & $90(11,8)$ & 83,3 & \\
\hline \multicolumn{4}{|l|}{ Angina } \\
\hline Não & $663(87,1)$ & 65,3 & \multirow{2}{*}{$<0,001^{\#}$} \\
\hline Sim & $98(12,9)$ & 83,7 & \\
\hline \multicolumn{4}{|l|}{ Diabetes } \\
\hline Não & $555(72,9)$ & 60,9 & \multirow{2}{*}{$<0,001^{\#}$} \\
\hline Sim & $206(27,1)$ & 85,9 & \\
\hline \multicolumn{4}{|c|}{ Doença cerebrovascular } \\
\hline Não & $642(84,4)$ & 64,6 & \multirow{2}{*}{$<0,001^{\#}$} \\
\hline Sim & $119(15,6)$ & 84 & \\
\hline \multicolumn{4}{|l|}{ Doenças mentais } \\
\hline Não & $475(62,4)$ & 65,1 & \multirow{2}{*}{$0,046^{\#}$} \\
\hline Sim & $286(37,6)$ & 72 & \\
\hline \multicolumn{4}{|l|}{ Doença pulmonar } \\
\hline Não & $624(82,0)$ & 67,3 & \multirow{2}{*}{$0,645^{\#}$} \\
\hline Sim & $137(18,0)$ & 69,3 & \\
\hline \multicolumn{4}{|l|}{ Doença hepática } \\
\hline Não & $710(93,3)$ & 67,6 & \multirow{2}{*}{$0,880^{\#}$} \\
\hline Sim & $51(6,7)$ & 68,6 & \\
\hline \multicolumn{4}{|l|}{ Doença renal } \\
\hline Não & $688(90,4)$ & 66,9 & \multirow{2}{*}{$0,141^{\#}$} \\
\hline Sim & $73(9,6)$ & 75,3 & \\
\hline \multicolumn{4}{|l|}{ Doença reumatológica } \\
\hline Não & $475(62,4)$ & 61 & \multirow{2}{*}{$0,233^{\#}$} \\
\hline Sim & $286(37,6)$ & 70,3 & \\
\hline
\end{tabular}

Fonte: primária.

Legenda: \#Teste Qui-Quadrado de Pearson. HAS = hipertensão arterial sistêmica; ICC = insuficiência cardíaca congestiva; IAM = infarto agudo do miocárdio.

Notas: o total de cada variável pode não atingir o n de 761 devido a perdas amostrais. 
Os medicamentos cardiovasculares mais relatados pelos idosos foram: hidroclorotiazida $(34,8 \%)$, captopril $(33,5 \%)$, sinvastatina $(26,1 \%)$, propranolol $(12,6 \%)$ e anlodipina $(11,2 \%)$. Na avaliação da classe farmacológica, segundo a ATC, as classes mais utilizadas foram os agentes que atuam sobre o sistema renina-angiotensina $(46,8 \%)$, os diuréti$\cos (42 \%)$, os hipolipemiantes $(26,9 \%)$ e os betabloqueadores $(24,7 \%)$ (Tabela 4 ).

Tabela 4 - Frequência de utilização de medicamentos por classe da ATC e dos medicamentos cardiovasculares mais utilizados

\begin{tabular}{l|r}
\hline \multicolumn{1}{c|}{ Classificação ATC } & $\begin{array}{c}\text { Frequência } \\
(\%)\end{array}$ \\
\hline Classes farmacológicas & \\
Agentes que atuam sobre o sistema \\
renina-angiotensina (C09) & 46,8 \\
Diuréticos (C03) & 42,0 \\
Hipolipemiantes (C10) & 26,9 \\
Betabloqueadores (C07) & 24,7 \\
Bloqueadores dos canais de cálcio & 14,2 \\
(C08) & 7,1 \\
Terapêutica cardíaca (C01) & 2,5 \\
Anti-hipertensivos (C02) & 0,1 \\
Vasodilatadores periféricos (C04) & 0,1 \\
Vasoprotetores (C05) & \\
Medicamentos cardiovasculares mais & \\
utilizados & 34,8 \\
Hidroclorotiazida (C03AA03) & 33,5 \\
Captopril (C09AA01) & 26,1 \\
Sinvastatina (C10AA01) & 12,6 \\
Propranolol (C07AA05) & 11,2 \\
Anlodipina (C08CA01) & 9,6 \\
Enalapril (C09AA02) & 6,6 \\
Furosemida (C03CA01) & 6,0 \\
Atenolol (C07AB03) & 5,3 \\
Metoprolol (C07AB02) & \\
\hline Fonte: primária &
\end{tabular}

Fonte: primária.

Nota: percentuais obtidos com base no total da amostra $(\mathrm{n}=761)$.
A Tabela 5 apresenta o modelo final da análise multivariada. As seguintes características mostraram uma associação significativa, de forma independente, com o uso de alguma medicação cardiovascular: sexo feminino $(\mathrm{RP}=1,14)$; cor negra $(\mathrm{RP}=1,10)$; consulta nos últimos seis meses $(\mathrm{RP}=1,34)$; HAS $(\mathrm{RP}=4,12)$; diabetes $(R P=1,08)$; e doença cerebrovascular $(\mathrm{RP}=1,10)$.

Tabela 5 - Modelo final da análise multivariada utilizando a regressão de Poisson para o desfecho de uso de medicamento cardiovascular por idosos da ESF de Porto Alegre

\begin{tabular}{l}
\multicolumn{2}{c}{ Variáv } \\
\hline Sexo \\
Masculino \\
Feminino \\
Cor \\
Branca \\
Parda \\
Negra \\
Outras \\
Consulta mé
\end{tabular}

Consulta médica

(últimos 6 meses)

Não

Sim

HAS

Não

Sim

Diabetes

Não

Sim

Doença cerebrovas-

cular

Não

Sim

Legendas: $\mathrm{RP}=$ razão de prevalência; $\mathrm{IC}=$ intervalo de confiança; HAS = hipertensão arterial sistêmica. 


\section{Discussão}

Este estudo avaliou a prevalência de uso de medicamentos cardiovasculares em uma população de idosos atendidos pelo SUS, identificando que $67,7 \%$ utilizavam pelo menos um desses medicamentos. $\mathrm{O}$ resultado está de acordo com outro estudo semelhante, publicado em 2007, com pacientes idosos da população geral de Porto Alegre, RS, que demonstrou uma prevalência de 62,9\% (SCHROETER et al., 2007). Esse é um dado relevante, considerando que, em outras pesquisas nacionais com a população idosa, a prevalência encontrada foi de 54,65\% no município de Santa Rosa, RS (FLORES; BENVEGNÚ, 2008), e de $55,42 \%$ em Rondonópolis, MT (GOULART et al., 2014). A maior prevalência encontrada no nosso estudo pode estar relacionada a um melhor acesso a diagnóstico e tratamento dessas doenças pelos idosos atendidos na ESF desse município.

Houve um claro predomínio do sexo feminino no uso de medicamentos cardiovasculares, que se manteve presente de forma independente na análise multivariada, corroborando os resultados encontrados em outros estudos nacionais (FLORES; BENVEGNÚ, 2008; GOULART et al., 2014; RIBAS; OLIVEIRA, 2014). Esse dado vem ao encontro da realidade atual da população idosa brasileira, na qual as mulheres são predominantes entre os idosos, como descrito pelo IBGE (2002), e também está de acordo com as VI Diretrizes Brasileiras de Hipertensão Arterial, que indicam que após os 50 anos de idade a prevalência global de hipertensão é maior entre as mulheres (SBC, 2010). As principais hipóteses para explicar esses achados são: as mulheres geralmente têm maior percepção da saúde e do autocuidado, buscam mais a assistência médica, apresentam maior adesão ao tratamento prescrito, assim como são contempladas por mais programas e políticas de saúde pública (BURNIER, 2006; CHOBANIAN et al., 2003).

Houve uma maior frequência de uso de medicamentos cardiovasculares na faixa dos 70-79 anos na análise bivariada. Esse dado é semelhante ao observado em um estudo conduzido nas cidades de Belo Horizonte e Rio de Janeiro (SILVA et al., 2012), mas difere de outros estudos que apontam para um maior uso de medicamentos por idosos mais jovens (60-69 anos) (COELHO FILHO; MARCOPITO; CASTELO, 2004; FLORES; BENVEGNÚ, 2008; FLORES; MENGUE, 2005). É possível que a maior frequência nessa faixa etária esteja associada ao fato de haver mais pessoas do sexo feminino, já que na análise multivariada essa associação desaparece.

Embora a análise bivariada não tenha demonstrado diferença significativa com relação à cor, na análise multivaria$\mathrm{da}$, a cor negra manteve-se independente para a associação do uso de medicamentos cardiovasculares. Estudos mostram que ser negro é um forte fator predisponente à HAS, ao desenvolvimento de hipertensão severa, a um maior risco de ataque cardíaco e à morte súbita. A pré-disposição em pessoas dessa cor parece 
estar relacionada a um defeito hereditário na captação celular de sódio e cálcio, o que facilitaria o aparecimento da HAS (SBC, 2010; BARRETO et al., 1993).

Indivíduos que realizaram consulta médica nos últimos seis meses apresentaram maior uso de medicamentos cardiovasculares, quando comparados com os que não realizaram consulta nesse período. Esse dado corrobora a associação do maior uso de medicamentos cardiovasculares com melhor acesso à saúde.

Os valores de IMC encontrados apontam uma diferença significativa ao comparar a amostra que utiliza medicamento cardiovascular com a amostra que não utiliza. Constatamos que pacientes classificados como baixo peso apresentam o menor uso de medicamentos cardiovasculares, enquanto, nas demais classificações, quanto maior o IMC maior a prevalência de uso desses medicamentos. Em alguns estudos (IRIBARREN et al., 1995; MANSON; WILLETT; STAMPFER, 1995), foi demonstrado que há um aumento dos índices de PA com o aumento do IMC e da idade (SUNDQUIST; WINKLEBY; PUDARIC, 2001), justificando um maior uso de medicamentos cardiovasculares por esses indivíduos. Os trabalhos de Cabrera e Jacob Filho (2001) e Zaitune et al. (2006) reforçam esses dados. Os estudos constataram que o IMC é um consistente índice antropométrico associado com uma maior prevalência de HAS em pacientes com valores de IMC mais elevados. Isso também é observado em estudos que identificam a relação da obesidade com doenças cardiovasculares (RODRÍGUEZ-HERNÁNDEZ et al., 2013; LÓPEZ-JARAMILLO et al., 2013; MEDEIROS et al., 2014).

Com relação às doenças autorreferidas, podemos observar, como esperado, uma associação entre as doenças relacionadas ao sistema cardiovascular e uma maior frequência de uso de medicamentos cardiovasculares. Além dessas, diabetes e doenças mentais também se relacionaram com um maior uso desses medicamentos. Provavelmente, esses achados demonstram que os fatores de risco cardiovasculares estão associados tanto com diabetes quanto com uma maior ocorrência de depressão e demência, principais doenças mentais que acometem idosos (JANI et al., 2014; GONTIJO et al., 2012; LICINIO; YILDIZ; WONG, 2002).

As doenças cardiovasculares correspondem às maiores causas de morbimortalidade entre os indivíduos acima de 65 anos, aumentando em número e gravidade com o avanço da idade (PANDOLFI; PIAZZOLLA; LOUZADA, 2010). Diversos trabalhos mostram, ainda, que os fármacos com ação sobre o sistema cardiovascular são os mais consumidos por idosos (FLORES; MENGUE, 2005; LOYOLA FILHO et al., 2005; MARIN et al., 2008; GALATO; SILVA; TIBURCIO, 2010).

Neste estudo, os subgrupos terapêuticos mais frequentemente utilizados foram os agentes que atuam sobre $o$ sistema renina-angiotensina, diuréticos, hipolipemiantes e betabloqueadores. Hidroclorotiazida, captopril e sinvasta- 
tina foram os medicamentos cardiovasculares mais citados pelos idosos. Esses achados são similares aos já descritos na literatura, exceto pela presença dos hipolipemiantes, que em outros estudos não aparecem em evidência (COELHO FILHO; MARCOPITO; CASTELO, 2004; FLORES; BENVEGNÚ, 2008; SCHROETER et al., 2007; FLORES; MENGUE, 2005). Como esse estudo foi conduzido com uma população de baixa renda, a frequência de uso desses medicamentos pode ser explicada pela presença dessas classes farmacológicas na Relação Municipal de Medicamentos (Remume) de Porto Alegre, que garante o fornecimento pelo SUS nessa cidade (REMUME, 2012).

Reconhecemos que este estudo apresenta limitações. Os resultados aplicam-se à população pertencente à ESF, ou seja, focada nos cuidados primários à saúde, e, portanto, podem não ser representativos do total da população brasileira. O padrão de consumo de medicamentos cardiovasculares é influenciado pelos medicamentos disponíveis no SUS. As variáveis autopercepção de saúde e doenças foram obtidas por meio de autorrelato. As limitações de um estudo transversal e descritivo devem ser consideradas, como a falta de controle de qualidade das informações fornecidas e a falta de verificações cruzadas entre as variáveis e a impossibilidade de estabelecer a temporalidade dos fatores associados. Os ajustes relativos foram aplicados no modelo final de análise multivariada para diminuir essas diferenças.

\section{Conclusão}

Em torno de $10 \%$ a $15 \%$ dos idosos que referiram uma doença cardiovascular não usam medicamento. Embora isso possa representar, em parte, uma falha na coleta dos dados pelas informações autorreferidas, esses dados demonstram que há idosos sem acesso a medicamentos.

Com a população idosa em constante crescimento, sendo ela a mais afetada por doenças cardiovasculares, o consumo desses fármacos pode afetar diretamente sua qualidade de vida. Acreditamos que informações provenientes de estudos populacionais, principalmente com a população assistida pelo SUS, vão auxiliar na formulação de estratégias e políticas públicas voltadas para uma assistência farmacêutica efetiva, com a participação do farmacêutico, que, acreditamos, deve estar presente em todos os níveis da assistência primária da saúde pública.

\section{Cardiovascular drugs: prevalence and associated factors in the elderly Family Health Strategy in Porto Alegre}

\section{Abstract}

The aim was investigate the prevalence of cardiovascular drugs use and its association with socio-demographic and clinical variables of elderly patients treated by Family Health Strategy through a cross-sectional study conducted in the municipality of Porto Alegre. Data was collected through a form that evaluated socio-demographic, economic aspects, self-reported diseases, and medication use. Specialized

RBCEH, Passo Fundo, v. 14, n. 2, p. 129-|44, maio/ago. 2017 
evaluations were conducted by a multidisciplinary team. Were evaluated 761 elders, with a $67.7 \%$ prevalence of cardiovascular drugs use. The characteristics that independently showed a significant association were: feminine gender, dark skin, examination in the past six months, systemic arterial hypertension, diabetes and cerebrovascular disease. Cardiovascular drugs and the most used classes, respectively, were: hydrochlorothiazide (34.8\%) and captopril $(33.5 \%)$; agents that act within the renin-angiotensin system (46.8\%) and diuretics $(42.0 \%)$. This data is important since the elderly population is mainly affected by cardiovascular disease and populational data helps in the creation of strategies and public policies aimed primarily at the elderly population treated by the public health system.

Keywords: Aged. Cardiovascular diseases. Family Health Strategy. Pharmaceuticals.

\section{Referências}

ALI RAZA, J.; MOVAHED, A. Use of cardiovascular medications in the elderly. International Journal of Cardiology, v. 85, n. 2-3, p. 203-215, 2002.

BARRETO, N. D. M. et al. Prevalência da hipertensão arterial nos indivíduos de raça negra. Arquivos Brasileiros de Medicina, Belo Horizonte, MG, v. 67, n. 6, p. 449-451, 1993.

BRASIL. Lei nº. 10.741, de 01 de outubro de 2003. Dispõe sobre o Estatuto do Idoso e dá outras providências. Diário Oficial da União, Brasília, DF, 03 out. 2003.

. Portaria do Gabinete do Ministro de Estado da Saúde de no 1395, de 9 de dezembro de 1999. Aprova a Política Nacional de Saúde do Idoso e dá outras providências. Diário Oficial da União, Brasília, DF, 13, 1999.
BURNIER, M. Medication adherence and persistence as the cornerstone of effective antihypertensive therapy. American Journal of Hypertension, v. 19, n. 11, p. 1190-1196, Nov. 2006.

CABRERA, M. A. S.; JACOB FILHO, W. Obesidade em idosos: prevalência, distribuição e associação com hábitos e co-morbidades. Arquivos Brasileiros de Endocrinologia \& Metabologia, São Paulo, SP, v. 45, n. 5, p. 494-501, out. 2001.

CHEN, Y. et al. China Kadoorie Biobank Study. Use of drug treatment for secondary prevention of cardiovascular disease in urban and rural communities of China: China Kadoorie Biobank Study of 0.5 million people. International Journal of Cardiology, v. 172, n. 1, p. 88-95, Mar. 2014.

CHOBANIAN, A. V. et al. Joint National Committee on Prevention, Detection, Evaluation, and Treatment of High Blood Pressure. National Heart, Lung, and Blood Institute; National High Blood Pressure Education Program Coordinating Committee. Seventh report of the Joint National Committee on Prevention, Detection, Evaluation, and Treatment of High Blood Pressure. Hypertension, v. 42, n. 6, p. 1206-1252, 2003.

DAMIÃO, D. S. et al. Avaliação do uso de medicamentos em pacientes idosos através de conceitos de farmacoepidemiologia e farmacovigilância em unidade básica de saúde do bairro CPA IV - Cuiabá, MT. COORTE - Revista Científica do Hospital Santa Rosa, Cuiabá, MT, n. 2, p. 46-49, jan./jun. 2011.

COELHO FILHO, J. M.; MARCOPITO, L. F.; CASTELO, A. Perfil de utilização de medicamentos por idosos em área urbana do Nordeste do Brasil. Revista de Saúde Pública, São Paulo, SP, v. 38, n. 4, p. 557-564, ago. 2004.

FLORES, L. M.; MENGUE, S. S. Uso de medicamentos por idosos em região do sul do Brasil. Revista de Saúde Pública da USP, São Paulo, SP, v. 39, n. 6, p. 924-929, dez. 2005. 
FLORES, V. B.; BENVEGNú, L. A. Perfil de utilização de medicamentos em idosos da zona urbana de Santa Rosa, Rio Grande do Sul, Brasil. Cadernos de Saúde Pública, Rio de Janeiro, RJ, v. 24, n. 6, p. 1439-1446, jun. 2008.

GALATO, D.; SILVA, E. S.; TIBURCIO, L. S. Estudo de utilização de medicamentos em idosos residentes em uma cidade do sul de Santa Catarina (Brasil): um olhar sobre a polimedicação. Revista Ciência \& Saúde Coletiva, Rio de Janeiro, RJ, v. 15, n. 6, p. 2899-2905, set. 2010.

GOMES, I. et al. The multidimensional study of the elderly in the family health strategy in Porto Alegre, Brazil (EMI-SUS). Pan American Journal of Aging Research, Porto Alegre, RS, v. 1, n. 1, p. 20-24, 2013.

GONTIJO, M. F. et al. Uso de anti-hipertensivos e antidiabéticos por idosos: inquérito em Belo Horizonte, Minas Gerais, Brasil. Cadernos de Saúde Pública, Rio de Janeiro, RJ, v. 28, n. 7, p. 1337-1346, jul. 2012.

GOULART, L. S. et al. Consumo de medicamentos por idosos de uma unidade básica de saúde de Rondonópolis/MT. Estudos Interdisciplinares sobre o Envelhecimento, Porto Alegre, RS, v. 19, n. 1, p. 79-94, abr. 2014.

INSTITUTO BRASILEIRO DE GEOGRAFIA E ESTATÍSTICA (IBGE). Perfil dos idosos responsáveis pelos domicílios no Brasil 2000. Rio de Janeiro: IBGE, 2002. Disponível em: $<$ http://biblioteca.ibge.gov.br/visualizacao/ livros/liv929.pdf>. Acesso em: 21 fev. 2015.

. Sinopse do Censo Demográfico 2010. Rio de Janeiro: IBGE, 2011. Disponível em: <http://www.ibge.gov.br/home/presidencia/ noticias/imprensa/ppts/0000000402.pdf>. Acesso em: 21 fev. 2015.

IRIBARREN, C. et al. Association of weight loss and weight fluctuation with mortality among Japanese American men. The New England Journal of Medicine, v. 333, p. 686692, 1995.
JACKSON, C. F.; WENGER, N. K. Cardiovascular Disease in the Elderly. Revista Española de Cardiología, v. 64, n. 8, p. 697712, 2011.

JANI, B. D. et al. Revisiting the J shaped curve, exploring the association between cardiovascular risk factors and concurrent depressive symptoms in patients with cardiometabolic disease: findings from a large cross-sectional study. BMC Cardiovascular Disorders, v. 14, p. 139-141, 2014.

SANTOS JÚNIOR, E. B.; OLIVEIRA, L. P. A. B.; SILVA, R. A. R. Doenças crônicas não transmissíveis e a capacidade funcional de idosos. Journal of Research: Fundamental Care Online, Rio de Janeiro, RJ, v. 6, n. 2, p. 516-524, abr./jun. 2014.

KALARIA, R. N. et al. Alzheimer's disease and vascular dementia in developing countries: prevalence, management, and risk factors. The Lancet Neurology, v. 7, n. 9, p. 812-826, 2008.

LICINIO, J.; YILDIZ, B.; WONG, M. L. Depression and cardiovascular disease: cooccurrence or shared genetic substrates? Molecular Psychiatry, v. 7, p. 1031-1032, 2002.

LÓPEZ-JARAMILLO, P. et al. Inflammation and hypertension: are there regional differences? International Journal of Hypertension, v. 2013, p. 492-504, 2013.

LOYOLA FILHO, A. I. et al. Estudo de base populacional sobre o consumo de medicamentos entre idosos: Projeto Bambuí. Cadernos de Saúde Pública, Rio de Janeiro, RJ, v. 21, n. 2, p. 545-553, mar./abr. 2005.

MAFRA, S. C. T. et al. O envelhecimento nas diferentes regiões do Brasil uma discussão a partir do censo demográfico 2010. In: CONGRESSO INTERNACIONAL DE ENVELHECIMENTO HUMANO, 3., Campina Grande. Anais... Campina Grande, 2013. p. 01-09. 
MANSON, J. E.; WILLETT, W. C.; STAMPFER, M. J. Body weight and mortality among women. The New England Journal of Medicine, v. 333, p. 677-685, 1995.

MARIN, M. J. S. et al. Caracterização do uso de medicamentos entre idosos de uma unidade do Programa Saúde da Família. Cadernos de Saúde Pública, Rio de Janeiro, RJ, v. 24, n. 7, p. 1545-1555, jul. 2008.

MEDEIROS, G. R. et al. Avaliação dos fatores de risco para doenças cardiovasculares relacionados à obesidade abdominal. Estudos, Goiânia, GO, v. 41, n. 3, p. 495-505, jul./ set. 2014.

PANDOLFI, M. B.; PIAZZOLLA, L. P.; LOUZADA, L. L. Prevalência de polifarmácia em idosos residentes em instituição de longa permanência de Brasília-DF. Brasília Médica, Brasília, DF, v. 47, n. 1, p. 53-58, 2010.

PERROTTI, T. C. et al. Tratamento farmacológico da hipertensão no idoso. Revista Brasileira de Hipertensão, Rio de janeiro, RJ, v. 14, n. 1, p. 37-41, jan./mar. 2007.

REMUME. Relação Municipal de Medicamentos 2012. Porto Alegre: Prefeitura Municipal de Porto Alegre, RS, 2012. Disponível em: <http://proweb.procempa.com.br/pmpa/ prefpoa/sms/usu_doc/remume_2012.pdf $>$. Acesso em: 8 out. 2015.

RIBAS, C.; OLIVEIRA, K. R. Perfil dos medicamentos prescritos para idosos em uma Unidade Básica de Saúde do município de Ijuí-RS. Revista Brasileira de Geriatria e Gerontologia, Rio de Janeiro, RJ, v. 17, n. 1, p. 99-114, jan./mar. 2014.

RODRÍGUEZ-HERNÁNDEZ, H. et al. Obesity and inflammation: epidemiology, risk factors, and markers of inflammation. International Journal of Endocrinology, v. 2013, p. 678-688, 2013.

SCHROETER, G. et al. Terapia anti-hipertensiva utilizada por pacientes idosos de Porto Alegre/RS, Brasil. Scientia Medica, Porto Alegre, RS, v. 17, n. 1, p. 14-19, jan./ mar. 2007.
SILVA, A. L. et al. Utilização de medicamentos por idosos brasileiros, de acordo com a faixa etária: um inquérito postal. Cadernos de Saúde Pública, Rio de Janeiro, RJ, v. 28, n. 6, p. 1033-1045, jun. 2012.

SOCIEDADE BRASILEIRA DE CARDIOLOGIA. VI Diretrizes Brasileiras de Hipertensão. Arquivos Brasileiros de Cardiologia, Rio de Janeiro, RJ, v. 95, supl. 1, p. 1-51, jul. 2010.

SUNDQUIST, J.; WINKLEBY, M. A.; PUDARIC, S. Cardiovascular disease risk factors among older black, Mexican-American, and white women and men: an analysis of NHANES III, 1988-1994. Journal of the American Geriatrics Society, v. 49, p. 109116, 2001.

VIACAVA, F. Acesso e uso de serviços de saúde pelos brasileiros: estudo realizado a partir dos dados da Pnad/IBGE analisa desigualdades geográficas e de renda e monitora o desempenho das políticas de saúde. Radis - Comunicação em Saúde, Rio de Janeiro, RJ, n. 96 , p. 12-19, ago. 2010.

WILLIAMS, B. The year in hypertension. Journal of the American College of Cardiology, v. 55, n. 1, p. 66-73, 2010.

WORLD HEALTH ORGANIZATION. Collaborating Centre for Drug Statistics Methodology. Anatomical-Therapeutic-Chemical (ATC) classification index. Genebra: WHO, 2015. Disponível em: <http://www.whocc.no/ atc_ddd_index/>. Acesso em: 11 maio 2015.

. Consultation on Obesity. Obesity: prevention and managing: the global epidemic. Report of a WHO Consultation on Obesity. Genebra: WHO, 2000. Disponível em: <http://www.who.int/nutrition/publications/obesity/WHO_TRS_894/en/>. Acesso em: 19 out. 2015.

Envelhecimento ativo: uma política de saúde. Brasília: WHO, 2005. Disponível em: $<$ https://bvsms.saude.gov.br/bvs/publicacoes/ envelhecimento_ativo.pdf $>$. Acesso em: 15 fev. 2015. 
YAZDANYAR, A.; NEWMAN, A. B. The burden of cardiovascular disease in the elderly: morbidity, mortality, and costs. Clinics in Geriatric Medicine, v. 25, n. 4, p. 563-584, 2009.

ZAITUNE, M. P. A. et al. Hipertensão arterial em idosos: prevalência, fatores associados e práticas de controle no Município de Campinas, São Paulo, Brasil. Cadernos de Saúde Pública, Rio de Janeiro, RJ, v. 22, n. 2, p. 285-294, fev. 2006. 